\title{
Comparative diagnostic performance of ultrasound shear wave elastography and magnetic resonance elastography for classifying fibrosis stage in adults with biopsy-proven nonalcoholic fatty liver disease
}

\author{
Yingzhen N. Zhang ${ }^{1} \cdot$ Kathryn J. Fowler ${ }^{1}$. Andrew S. Boehringer ${ }^{1}$. Vivian Montes ${ }^{1}$. Alexandra N. Schlein ${ }^{1}$. \\ Yesenia Covarrubias ${ }^{1} \cdot$ Tanya Wolfson $^{1}$. Cheng W. Hong ${ }^{1} \cdot$ Mark A. Valasek $^{2} \cdot$ Michael P. Andre $^{3} \cdot$ Rohit Loomba $^{4,5}$. \\ Claude B. Sirlin ${ }^{1}$ (1)
}

Received: 17 March 2021 / Revised: 7 September 2021 / Accepted: 9 October 2021 / Published online: 2 December 2021

(C) The Author(s) 2021

\begin{abstract}
Objectives To compare the diagnostic accuracy of US shear wave elastography (SWE) and magnetic resonance elastography (MRE) for classifying fibrosis stage in patients with nonalcoholic fatty liver disease (NAFLD).

Methods Patients from a prospective single-center cohort with clinical liver biopsy for known or suspected NAFLD underwent contemporaneous SWE and MRE. AUCs for classifying biopsy-determined liver fibrosis stages $\geq 1, \geq 2, \geq 3$, and $=4$, and their respective performance parameters at cutoffs providing $\geq 90 \%$ sensitivity or specificity were compared between SWE and MRE.

Results In total, 100 patients (mean age, $51.8 \pm 12.9$ years; $46 \%$ males; mean BMI $31.6 \pm 4.7 \mathrm{~kg} / \mathrm{m}^{2}$ ) with fibrosis stage distribution (stage $0 / 1 / 2 / 3 / 4$ ) of $43,36,5,10$, and $6 \%$, respectively, were included. AUCs (and 95\% CIs) for SWE and MRE were $0.65(0.54-0.76)$ and $0.81(0.72-0.89), 0.81(0.71-0.91)$ and $0.94(0.89-1.00), 0.85(0.74-0.96)$ and $0.95(0.89-1.00)$, and 0.91 (0.79-1.00) and 0.92 (0.83-1.00), for detecting fibrosis stage $\geq 1, \geq 2, \geq 3$, and $=4$, respectively. The differences were significant for detecting fibrosis stage $\geq 1$ and $\geq 2(p<0.01)$ but not otherwise. At $\geq 90 \%$ sensitivity cutoff, MRE yielded higher specificity than SWE at diagnosing fibrosis stage $\geq 1, \geq 2$, and $\geq 3$. At $\geq 90 \%$ specificity cutoff, MRE yielded higher sensitivity than SWE at diagnosing fibrosis stage $\geq 1$ and $\geq 2$.

Conclusions In adults with NAFLD, MRE was more accurate than SWE in diagnosing stage $\geq 1$ and $\geq 2$ fibrosis, but not stage $\geq 3$ or 4 fibrosis.

Key Points

- For detecting any fibrosis or mild fibrosis, MR elastography was significantly more accurate than shear wave elastography.

- For detecting advanced fibrosis and cirrhosis, MRE and SWE did not differ significantly in accuracy.

- For excluding advanced fibrosis and potentially ruling out the need for biopsy, SWE and MRE did not differ significantly in negative predictive value.

- Neither SWE nor MRE had sufficiently high positive predictive value to rule in advanced fibrosis.
\end{abstract}

Claude B. Sirlin

csirlin@health.ucsd.edu

1 Liver Imaging Group, Altman Clinical and Translational Research Institute, Department of Radiology, University of California, San Diego, 9452 Medical Center Drive, La Jolla, CA 92037, USA

2 Department of Pathology, University of California, San Diego, 9452 Medical Center Drive, La Jolla, CA 92037, USA
3 Department of Radiology, University of California, San Diego, 9452 Medical Center Drive, La Jolla, CA 92037, USA

4 NAFLD Research Center, Department of Medicine, University of California, San Diego, 9452 Medical Center Drive, La Jolla, CA 92037, USA

5 Division of Gastroenterology, Department of Medicine, University of California, San Diego, 9452 Medical Center Drive, La Jolla, CA 92037, USA 
Keywords Nonalcoholic fatty liver disease $\cdot$ Elasticity imaging techniques $\cdot$ Sonoelastography $\cdot$ Magnetic resonance elastography

\section{Abbreviations}

2D Two-dimensional

AASLD American Association for the Study of Liver Diseases

BMI Body mass index

CI Confidence interval

CSE Confounder-corrected chemical-shift-encoded

GRE Gradient-recalled-echo

IQR Interquartile range

MRE Magnetic resonance elastography

NAFLD Nonalcoholic fatty liver disease

NASH Nonalcoholic steatohepatitis

PDFF Proton-density fat fraction

pSWE Point shear wave elastography

SWE Ultrasound shear wave elastography

SWS Shear wave speed

TE Transient elastography

\section{Introduction}

With an estimated global prevalence of $25 \%$, nonalcoholic fatty liver disease (NAFLD) is the most common chronic liver disease worldwide [1]. NAFLD comprises both nonalcoholic fatty liver and nonalcoholic steatohepatitis (NASH), the latter of which is characterized by hepatocellular injury, inflammation, and higher potential for developing fibrosis. The severity of liver fibrosis in NASH strongly predicts long-term outcomes including liver transplantation and overall mortality [2]. Left untreated, liver fibrosis may progress to cirrhosis, conferring increased risk of hepatocellular carcinoma and liver-related mortality. Early therapeutic intervention in patients with NASH-related fibrosis may stabilize or even reverse fibrosis [3, 4]. Accurate diagnosis and staging of liver fibrosis enable risk stratification, monitoring for progression, and targeting interventions in these patients.

Histology is the current clinical standard for assessing fibrosis stage but liver biopsy is invasive, costly, and associated with non-negligible complication risk [5]. These drawbacks make histology impractical for screening patients with NAFLD, and noninvasive methods for assessing liver fibrosis are needed. To address this need, several elastography techniques have been developed for detecting and staging fibrosis noninvasively. Two-dimensional shear wave elastography (SWE, also known as sonoelastography), an advanced ultrasound-based technique, has comparable to superior accuracy for diagnosing fibrosis in NAFLD patients compared to older ultrasound-based techniques such as transient elastography (TE) and point shear wave elastography (pSWE) [6, 7]. Magnetic resonance elastography (MRE), an MR-based technique, has shown excellent performance for diagnosing and staging fibrosis in NAFLD patients $[8,9]$. While comparative evidence is accumulating, the optimal selection of SWE versus MRE remains unclear in the context of NAFLD. NAFLD may pose specific challenges to noninvasive techniques due to its association with steatosis (which alters sonographic echoes and MR signals) and obesity (which increases the abdominal wall thickness leading to potentially less reliable results). Two recent studies comparing the diagnostic performance of MRE and SWE showed either significant difference at staging cirrhosis only or no difference between the two methods in cohorts where the majority had at least significant fibrosis (stage $\geq 2$ ) $[10,11]$. These studies provide important comparative data on the performance of these methods in assessing fibrosis in patient populations with relatively advanced fibrosis stage distributions. However, studies are lacking that compare the diagnostic accuracy of MRE and SWE at staging the full spectrum of liver fibrosis severity in patients with NAFLD, particularly those who might have earlier stages of fibrosis, using histopathology as the reference standard.

The purpose of this study is to compare the diagnostic performance of SWE versus MRE for staging fibrosis in a well-characterized cohort of American adults with suspected or known NAFLD using histopathology as the reference standard. Secondarily, we explored the impact of obesity and hepatic steatosis on performance.

\section{Materials and methods}

\section{Study design}

This is a cross-sectional study of a prospectively recruited cohort of patients with known or suspected NAFLD who underwent liver biopsy for clinical care and contemporaneous SWE and MRE for research within 180 days of liver biopsy between July 2016 and June 2019. Confoundercorrected chemical-shift-encoded (CSE)-MRI was performed as part of the MRE exam in order to estimate proton-density fat fraction (PDFF), which was used to stratify the cohort in the exploratory analyses. This study was approved by the Institutional Review Board and is compliant with the Health Insurance Portability and Accountability Act. 
Study participants were recruited at the University of California, San Diego (UCSD), NAFLD Research Center. The screening process consisted of a standardized clinical evaluation which included a detailed physical examination, biochemical profiling, and an alcohol history assessment performed using the Alcohol Use Disorders Identification Test and Skinner Lifetime Drinking questionnaires. Eligible participants provided written informed consent to undergo SWE and MRE. Participants were instructed to fast for at least $8 \mathrm{~h}$ prior to SWE and MRE exams.

\section{Histologic analysis}

For this research, a single experienced hepatopathologist (M.A.V., > 10 years of experience) reviewed the clinically obtained biopsy specimen and scored the histologic features using the NASH Clinical Research Network histologic scoring system [12]. Fibrosis was scored from 0 to 4 , steatosis from 0 to 3 , lobular inflammation from 0 to 3 , and hepatocellular ballooning from 0 to 2 .

Details of eligibility criteria, clinical assessments, liver biopsy protocol, and histology interpretation are available as Supplemental Methods.

\section{SWE exam}

SWE exams were performed on a clinical ultrasound system (GE Logiq E9, GE Healthcare) provided by GE for this research through an equipment loan agreement. The ultrasound system was equipped with the transducer and software required for SWE.

One of four certified diagnostic medical sonographers (each with $>10$ years of clinical experience in abdominal US exams and at least 1 year of research experience in SWE) performed SWE using a convex transducer (C1-6). Sonographers were scheduled for each exam based on availability.

For SWE, participants were imaged in the dorsal decubitus position with the right arm fully abducted to facilitate a right intercostal approach. The transducer was oriented perpendicular to the liver capsule to optimize the acoustic window. Then, SWE was activated and, once a real-time colorized stiffness map of the right liver parenchyma had stabilized during an 8-10-s breath hold at shallow expiration, the sonographer recorded the stiffness map with a button press. The sonographer then placed a circular ROI at least $1 \mathrm{~cm}$ below the liver capsule but no more than $8 \mathrm{~cm}$ from the skin surface that overlaid as much of the homogeneous color map as possible while avoiding large blood vessels, portal tracts, and rib shadowing. The mean and standard deviation of shear wave speed values within the ROI were recorded.
The above steps were repeated until 10 sequential shear wave speed (SWS) measurements were acquired per participant (out of a maximum of 20 attempts), as recommended by the manufacturer. A study was considered adequate if the IQR for the 10 measurements was less than $30 \%$ of the median $(\mathrm{IQR} /$ median $<0.30)[13,14]$. The entire SWE exam lasted about $10 \mathrm{~min}$.

\section{MR exam: MRE and chemical-shift-encoded MRI}

MR exams were performed using a 3-T research scanner with a 60-cm bore (GE Discovery MR750; GE Healthcare) and a 32-channel torso radiofrequency coil array. The scanner was fitted with MRE hardware and software licensed for research (Resoundant) [15, 16]. The entire MR exam including participant positioning, MRE driver placement, and MRE and CSE-MRI acquisition lasted about $20 \mathrm{~min}$.

\section{MRE sequence and analysis}

An active acoustic driver set to the standard frequency of $60-\mathrm{Hz}$ delivered vibrations via a passive pneumatic driver that was centered over the liver and secured snugly to the abdominal wall by an elastic band. A two-dimensional (2D) gradient-recalled-echo (GRE) MRE sequence modified with bipolar motion encoding gradients synchronized to the applied vibration imaged the shear wave displacement. Four 10-mm contiguous axial slices were acquired through the widest transverse section of the liver, each with a 16-s breath hold performed at relaxed end-expiration. Acquisition parameters are listed in Supplemental Methods. Using MRE reconstruction software, the MR scanner automatically processed the wave images into cross-sectional 2D shearstiffness maps; unreliable pixels (goodness-of-fit $R^{2}<95 \%$ ) were cross-hatched to exclude them from analysis [17].

One of two trained image analysts (each with $>1$ year of experience in MRE analysis) downloaded the raw and processed MRE data for offline analysis. Using MRE analysis software ("MRE Quant", Resoundant), the analyst manually drew free-form ROIs on portions of the right hepatic lobe on the wave images while avoiding the liver edge (outer $1 \mathrm{~cm}$ ), major vessels, and areas of nonplanar or low amplitude wave propagation $[4,18,19]$. The ROIs were drawn on all four slices and colocalized to the shear-stiffness maps. The mean of liver stiffness in the ROIs (shear stiffness, in kilopascals) and cumulative ROI size over four slices (in pixels) were automatically reported by the software. An MRE exam was considered adequate if the total number of pixels over four slices acquired in a participant was greater than or equal to 700 pixels [20]. 


\section{Chemical-shift-encoded MRI acquisition and analysis}

A 2D multi-echo spoiled gradient-recalled-echo sequence with magnitude reconstruction was performed through the entire liver. Using a previously described custom algorithm, the MR scanner automatically processed the source images into cross-sectional PDFF maps [21-24], which were analyzed offline to calculate mean liver PDFF values. Acquisition and analysis details are described in Supplemental Methods.

\section{Blinding}

The pathologist was blinded to imaging data. Sonographers were blinded to clinical, histological, and MRI data. MR analysts were blinded to clinical, histological, and ultrasound data.

\section{Statistical analyses}

Statistical analysis was performed by a postdoctoral fellow (Y.N.Z., 2 years of experience) under the supervision of a biostatistician (T.W., with 25 years of experience) using "R" statistical computing software (R version 3.4.2 [2016]; R Foundation for Statistical Computing).

Sample size was based on feasibility. The target enrollment was set to $\geq 100$ participants who complied with the study protocol and completed SWE and MRE.

\section{Diagnostic performance}

Analyses of diagnostic performance were performed in participants in whom both SWE and MRE were adequate, as defined earlier. Spearman's correlation was used to evaluate the relationship between SWE, MRE, and fibrosis stages.

ROCs and AUCs with DeLong 95\% confidence intervals (CIs) were computed for SWE and MRE for classifying each dichotomized fibrosis stage. AUCs were compared using the DeLong test for dependent ROCs. The shear wave speed cutoffs (SWE) or stiffness cutoffs (MRE) providing at least $90 \%$ sensitivity or at least 90\% specificity for each dichotomization were identified. Performance parameters at those cutoffs were compared using McNemar's test for paired proportions. The Bonferroni correction was applied to each grouped comparison of AUC, sensitivity at $90 \%$ specificity, and specificity at $90 \%$ sensitivity. A $p$ value less than 0.05 (or individual $p$ value $<0.05 / 3=0.017$ after the Bonferroni correction) was considered statistically significant. We chose a priori not to formally compare additional performance metrics (PPV, NPV, total accuracy) to reduce the number of comparisons.

\section{Exploratory analyses}

To evaluate the impact of obesity and steatosis on both techniques, the above analyses were repeated separately in obese $(\mathrm{BMI} \geq 30)$ and nonobese $(\mathrm{BMI}<30)$ participants and in those with none-to-mild and moderate-to-severe steatosis as determined noninvasively by published PDFF cutoffs (none-to-mild: PDFF $<17.43 \%$; moderate-to-severe: $\mathrm{PDFF} \geq 17.43 \%)$ [21].

\section{Results}

\section{Participants}

Between July 2016 and June 2019, 118 patients with liver biopsies to evaluate known or suspected NAFLD met inclusion criteria, of whom 18 were excluded (Fig. 1), leaving 100 participants in the final analysis.

Table 1 summarizes the baseline demographic, biochemical, histological, and imaging data for these 100 participants. Sixty-four participants $(64 \%)$ were obese (BMI $\geq 30.0)$. The median PDFF was $14.2 \%$. Median time intervals were 27 days between SWE and biopsy, 28 days between MRE and biopsy, and 0 days between SWE and MRE. The average ( \pm standard deviation [SD]) of biopsy size and number of portal triads were $21.8( \pm 7.3) \mathrm{mm}$ and $14.5( \pm 3.7)$, respectively. In total, 43, 36, 5, 10, and 6 had fibrosis stages 0,1 , 2,3 , and 4 , respectively.

\section{Diagnostic performance}

Mean shear wave speed and stiffness values are shown in Fig. 2. Mean shear wave speed and stiffness values increased monotonically with fibrosis stages (Spearman's correlation coefficient for shear wave speed values and fibrosis stages is $0.392(p<0.01)$, and for stiffness values and fibrosis stages is $0.654(p<0.01)$. Representative SWE and MRE images are shown in Fig. 3. The mean $( \pm$ SD) area of the captured SWE ROIs was $1.19( \pm 0.39) \mathrm{cm}^{2}$, and the mean $( \pm \mathrm{SD})$ cumulative ROI size over 4 slices of MRE for each participant was $3350( \pm 1498)$ pixels $\left(469 \mathrm{~cm}^{2} \pm 210 \mathrm{~cm}^{2}\right)$. 
The AUCs of MRE were significantly higher than those of SWE for diagnosing any fibrosis (0.81 [95\% CI: 0.72-0.89] vs. 0.65 [95\% CI: $0.54-0.76$ ] $p=0.005)$ and stage $\geq 2$ fibrosis (0.94 [95\% CI: 0.89-1.00] vs. 0.81 [95\% CI: 0.71-0.91], $p=0.009$ ). The AUC point estimates of MRE were nominally higher than those of SWE for stage $\geq 3$ and stage $=4$ fibrosis, but the differences were not significant (Table 2).

Cutoffs and performance parameters for the classification of dichotomized fibrosis stages given predefined sensitivity or specificity $\geq 90 \%$ are summarized in Tables 3 and 4 .

At sensitivity of at least $90 \%$, the SWE cutoffs were 1.27 , $1.49,1.46$, and $1.59 \mathrm{~m} / \mathrm{s}$ for stage $\geq 1$ fibrosis, stage $\geq 2$ fibrosis, stage $\geq 3$ fibrosis, and stage 4 fibrosis, respectively; the MRE cutoffs were $2.01,2.77,2.77$, and $2.77 \mathrm{kPa}$, respectively. MRE had higher specificity than SWE for all stages of fibrosis, and the difference was significant for stage $\geq 1, \geq 2$, and $\geq 3$ ( $p<0.001)$. The point estimate for PPV was higher for MRE than for SWE for all stages of fibrosis among this particular cohort, though formal statistical comparisons were not performed.
At specificity of at least $90 \%$, the SWE cutoffs were 1.75 , $1.79,1.78$, and $1.81 \mathrm{~m} / \mathrm{s}$ for stage $\geq 1$ fibrosis, stage $\geq 2$ fibrosis, stage $\geq 3$ fibrosis, and stage 4 fibrosis, respectively; the MRE cutoffs were 2.60, 3.06, 3.17, and $3.42 \mathrm{kPa}$, respectively. MRE had higher sensitivity than SWE for all stages of fibrosis, and the difference was significant for fibrosis stages $\geq 1$ and $\geq 2(p \leq 0.01)$. The point estimate for NPV was higher for MRE than for SWE for all stages of fibrosis except cirrhosis (stage 4) among this particular cohort, though formal statistical comparisons were not performed.

\section{Exploratory analyses}

In stratified analysis of obese $(n=64)$ and nonobese $(n=36)$ participants, MRE was superior to SWE at diagnosing stage $\geq 1$ fibrosis among obese participants $(p=0.008)$. In stratified analysis of participants with none-to-mild steatosis using PDFF cutoff $<17.43 \%(n=68)$ and moderate-tosevere steatosis using PDFF cutoff $\geq 17.43 \%$ ( $n=32)$, MRE was superior to SWE at diagnosing stage $\geq 2$ fibrosis among
Fig. 1 Flowchart of participant selection. NAFLD, nonalcoholic fatty liver disease; $N A S H$, nonalcoholic steatohepatitis; $S W E$, shear wave elastography; $M R E$, magnetic resonance elastography

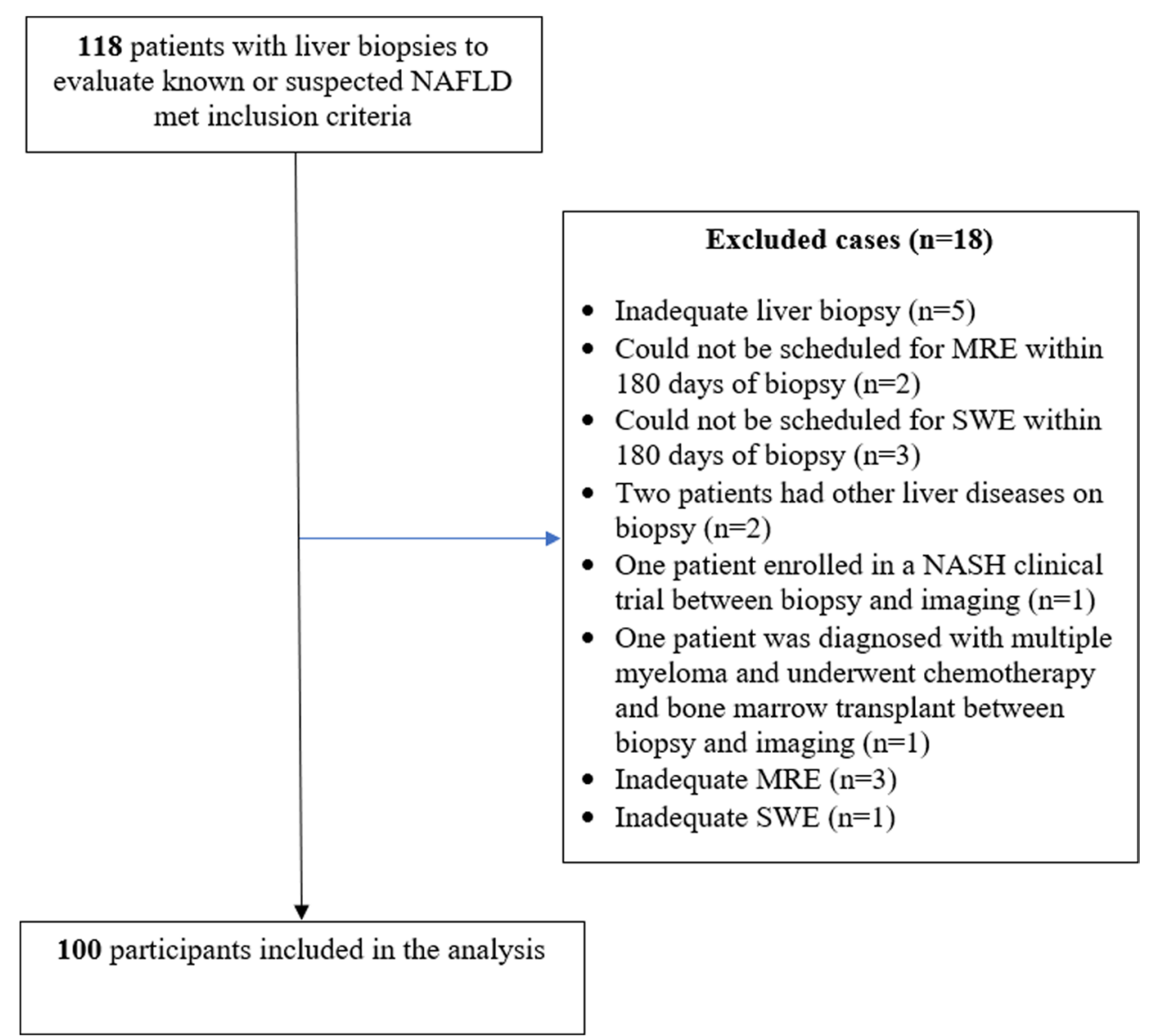


Table 1 Demographic, biochemical, histological, and imaging characteristics of study participants

\begin{tabular}{|c|c|}
\hline Characteristic & Values \\
\hline \multicolumn{2}{|c|}{ Demographic, anthropometric, biochemical, and imaging data $(n=100)$} \\
\hline \multicolumn{2}{|l|}{ Participant sex } \\
\hline Male & $46(46 \%)$ \\
\hline Female & $54(54 \%)$ \\
\hline Mean age (year), mean \pm SD [range] & $51.8 \pm 12.9[25-78]$ \\
\hline Mean body mass index $\left(\mathrm{kg} / \mathrm{m}^{2}\right)$, mean $\pm \mathrm{SD}$ [range] & $31.6 \pm 4.7[21.5-43.3]$ \\
\hline \multicolumn{2}{|l|}{ Self-reported race } \\
\hline Black or African American & $3(3 \%)$ \\
\hline Asian & $16(16 \%)$ \\
\hline White & $55(55 \%)$ \\
\hline Other & $26(26 \%)$ \\
\hline \multicolumn{2}{|l|}{ Self-reported ethnicity } \\
\hline Hispanic & $33(33 \%)$ \\
\hline Non-Hispanic & $66(66 \%)$ \\
\hline Declined to state & $1(1 \%)$ \\
\hline \multicolumn{2}{|l|}{ Biochemical profile, mean \pm SD } \\
\hline Aspartate aminotransferase level (U/L) & $43.7 \pm 18.6$ \\
\hline Alanine aminotransferase level (U/L) & $64.7 \pm 36.9$ \\
\hline Alkaline phosphatase level (IU/L) & $83.2 \pm 20.1$ \\
\hline Total bilirubin $(\mathrm{mg} / \mathrm{dL})$ & $0.5 \pm 0.2$ \\
\hline Albumin level (g/dL) & $4.4 \pm 0.7$ \\
\hline Glucose (mg/dL) & $116.0 \pm 32.9$ \\
\hline Platelet count (billion/L) & $270.2 \pm 71.1$ \\
\hline Prothrombin time (s) & $11.1 \pm 0.7$ \\
\hline International normalized ratio & $1.0 \pm 0.1$ \\
\hline \multicolumn{2}{|l|}{ Biopsy sample, mean \pm SD } \\
\hline Sample length $(\mathrm{mm})$ & $21.8 \pm 7.3$ \\
\hline No. of portal tracts & $14.5 \pm 3.7$ \\
\hline \multicolumn{2}{|l|}{ Histology, $n(\%)$} \\
\hline \multicolumn{2}{|l|}{ Fibrosis } \\
\hline 0 (no fibrosis) & $43(43 \%)$ \\
\hline 1 (perisinusoidal or periportal) & $36(36 \%)$ \\
\hline 2 (perisinusoidal and periportal) & $5(5 \%)$ \\
\hline 3 (bridging fibrosis) & $10(10 \%)$ \\
\hline 4 (cirrhosis) & $6(6 \%)$ \\
\hline \multicolumn{2}{|l|}{ Steatosis } \\
\hline $0(<5 \%$ hepatocytes $)$ & $6(6 \%)$ \\
\hline 1 (5-33\% hepatocytes) & $43(43 \%)$ \\
\hline 2 (33-66\% hepatocytes) & $40(40 \%)$ \\
\hline 3 (>66\% hepatocytes) & $11(11 \%)$ \\
\hline \multicolumn{2}{|l|}{ Lobular inflammation } \\
\hline 0 (no foci) & $7(7 \%)$ \\
\hline $1(<2$ foci per $200 \times$ field $)$ & $68(68 \%)$ \\
\hline $2(2-4$ foci per $200 \times$ field $)$ & $19(19 \%)$ \\
\hline 3 (> 4 foci per $200 \times$ field) & $6(6 \%)$ \\
\hline \multicolumn{2}{|l|}{ Ballooning } \\
\hline 0 (no ballooned cells) & $51(51 \%)$ \\
\hline 1 (few ballooned cells) & $45(45 \%)$ \\
\hline 2 (many ballooned cells or prominent ballooning) & $4(4 \%)$ \\
\hline \multicolumn{2}{|l|}{ Imaging } \\
\hline $\mathrm{SWE}, \mathrm{m} / \mathrm{s}$, mean $\pm \mathrm{SD}$ & $1.5 \pm 0.2$ \\
\hline
\end{tabular}


Table 1 (continued)

\begin{tabular}{ll}
\hline Characteristic & Values \\
\hline MRE, $\mathrm{kPa}$, mean $\pm \mathrm{SD}$ & $2.6 \pm 0.9$ \\
MRI-PDFF-per protocol, \%, mean (median) $\pm \mathrm{SD}$ & $13.9(14.2) \pm 8.1$ \\
SWE to biopsy time interval, days, mean (median) $\pm \mathrm{SD}$ & $37.6(27) \pm 34.8$ \\
MRE to biopsy time interval, days, mean (median) $\pm \mathrm{SD}$ & $33.3(28) \pm 31.7$ \\
SWE to MRE time interval, days, mean (median) $\pm \mathrm{SD}$ & $15.3(0) \pm 26.9$ \\
\hline
\end{tabular}

$S W E$ shear wave elastography, $M R E$ magnetic resonance elastography, $\mathrm{m} / \mathrm{s}$ meters per second, $\mathrm{kPa}$ kilopascals

participants with none-to-mild steatosis $(p=0.009)$, and at diagnosing stage $\geq 1$ fibrosis among participants with moderate-to-severe steatosis $(p=0.024)$.

\section{Discussion}

Noninvasive imaging methods for estimating fibrosis in NAFLD patients have been suggested both for initial detection and staging and for longitudinal monitoring, a scenario in which invasive tests like biopsy are not feasible. Patients with NAFLD pose several challenges (e.g., obesity, steatosis) that may impact imaging study performance. Hence, the optimal test or combination of tests has yet to be defined. Our study aimed to compare MRE and SWE against histological reference standard in a NAFLD population. While MRE was significantly more accurate than SWE for diagnosing lower stages of fibrosis (stage $\geq 1$ and $\geq 2$ ), the two techniques did not differ significantly at higher stages of fibrosis (stage $\geq 3$ and $=4$ ). In exploratory analyses, MRE also showed a trend towards better performance than SWE in all participant subgroups regardless of the presence of obesity or the severity of steatosis, though the differences between subgroups were sometimes significant only in the lower fibrosis stages.

Our study is the first to detect a significant difference in performance between SWE and MRE at diagnosing lower stages of fibrosis. A previous study by Furlan et al.on American adults with NAFLD examined the diagnostic performance of SWE and MRE at detecting significant fibrosis (stage $\geq 2$ ) and advanced fibrosis (stage $\geq 3$ ) and did not find a statistically significant difference, while a recent study by Imajo et al. on Japanese adults with NAFLD examined the diagnostic performance of SWE and MRE at detecting the full spectrum of fibrosis and found that MRE offered superior performance at staging cirrhosis only $[10,11]$. The small number of participants in both studies who had no liver fibrosis ( 1 in Furlan et al. and 9 in Imajo et al. had no liver fibrosis) may have limited their statistical power for comparing the diagnostic performance of SWE and MRE for detecting any fibrosis. Similarly, the relatively small number of participants in both studies who had mild disease (fibrosis stage <2) (18 in Furlan et al. and 59 in Imajo et al., compared to 79 in our study) may have reduced the power to detect differences in performance at diagnosing earlier stages of fibrosis. At advanced fibrosis (stage $\geq 3$ ), we-like Furlan et al.and Imajo et al.- - found that there is no statistically significant difference in performance between SWE and MRE. Conversely, the small number of participants in our study with cirrhosis (6 out of 100) likely reduced our power to detect differences in performance for diagnosing cirrhosis, and may explain why our results do not replicate the finding by Imajo et al. that MRE is superior to SWE for diagnosing cirrhosis. Despite the small number of participants with cirrhosis, our overall cohort was comparatively large, which allowed for exploratory analysis of NAFLD patients stratified by fibrosis severity and obesity, two potential confounders for noninvasive techniques.

Compared to published studies on the diagnostic performance of SWE for fibrosis staging in NAFLD patients, we found lower diagnostic accuracy as assessed with AUCs [7, 10, 25-27]. Differences in stage distribution may account in part for the discrepancy. A majority of participants $(58.3 \%$ to $78.4 \%)$ in published studies had stage $\geq 2$ fibrosis compared to a minority $(21 \%)$ in our cohort. The higher proportion of patients with more severe fibrosis in published studies is expected to increase the observed AUC, since greater separation between shear wave speed or shear stiffness values are observed at higher fibrosis stages [28]. Compared to study cohorts that skew towards the more severe spectrum of liver fibrosis, our results may be most applicable to the outpatient NAFLD hepatology clinic from which we enrolled our participants.

The diagnostic performance of MRE across all dichotomized fibrosis stages in our study was consistent with prior studies on NAFLD patients and overweight-to-obese patients, which included patients with similar fibrosis stage distribution $[8,9,29]$. We intentionally reported two sets of cutoff values for MRE and SWE — one set that would yield at least $90 \%$ sensitivity and one set that would yield at least $90 \%$ specificityfor each dichotomized fibrosis stage instead of the Youden index. While the Youden index maximizes the combination of sensitivity and specificity for a particular test, it is not as helpful in informing clinical application and interpretation. 
Fig. 2 Distribution of shear wave speed measurements by shear wave elastography (a) and liver stiffness measurements by magnetic resonance elastography (b) stratified by biopsy-determined fibrosis stage (Nonalcoholic Steatohepatitis Clinical Research Network) a

2D SWE on fibrosis stage

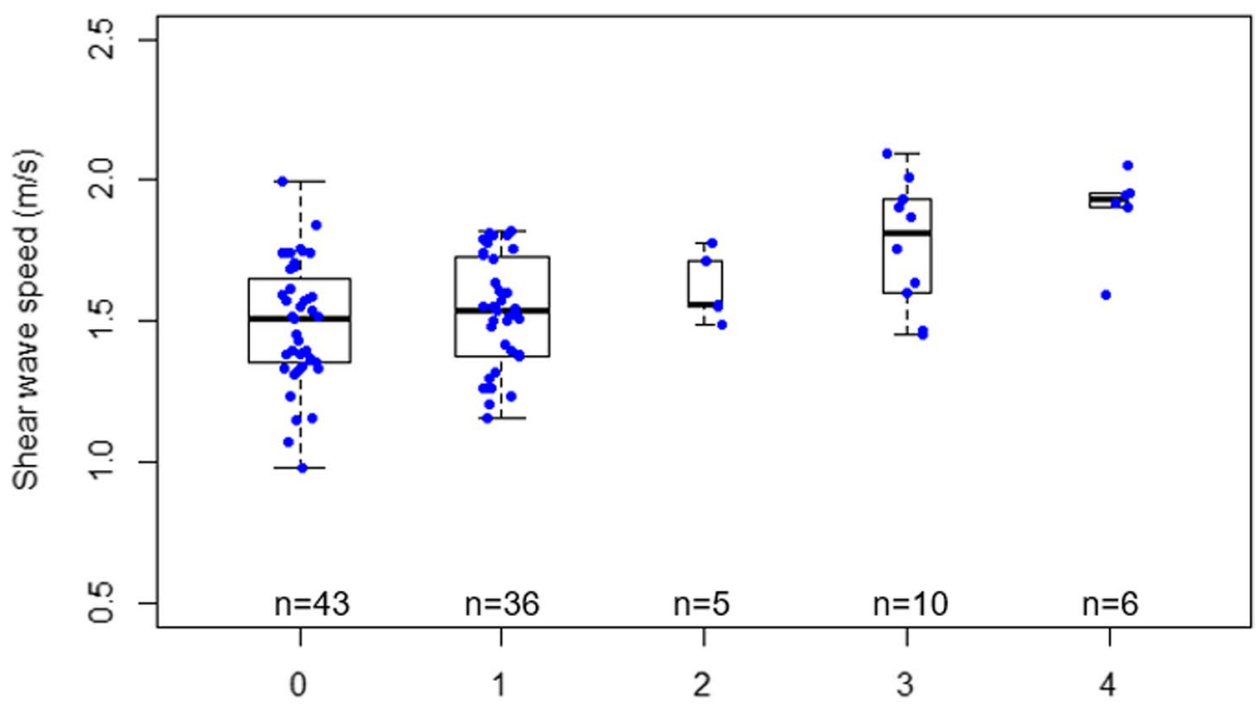

Fibrosis stage on histology

b 2D MRE on fibrosis stage

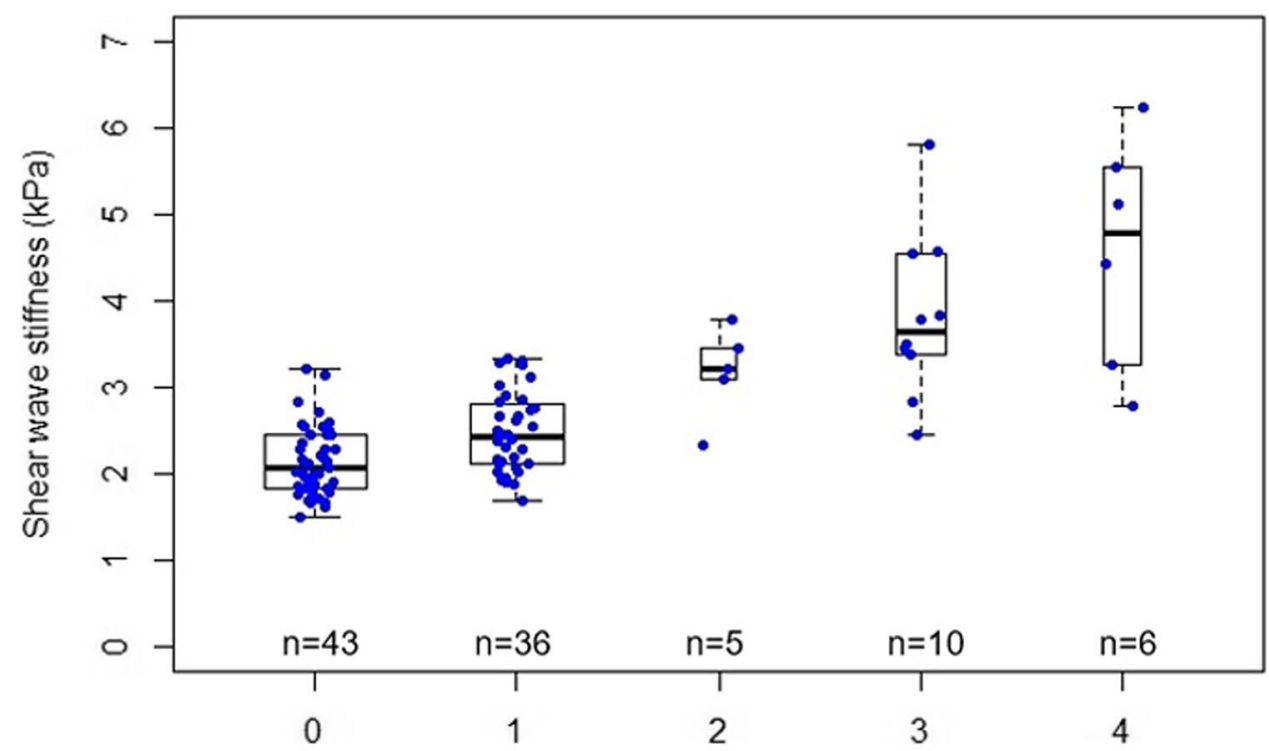

Fibrosis stage on histology
For instance, recent guidelines from the American Association for the Study of Liver Diseases (AASLD) suggest the use of noninvasive tests to detect patients with high likelihood of advanced stage fibrosis-i.e., those patients who may have the greatest benefit-to-risk ratio for biopsy [30]. This context of use requires high sensitivity and NPV to rule out fibrosis in order to appropriately direct biopsy to those at high risk. For this purpose, SWE and MRE did not differ in performance:
SWE can accurately exclude stage $\geq 3$ fibrosis with sensitivity of $94-100 \%$ and NPV of $97-100 \%$ while MRE can do so with sensitivity of $94-100 \%$ and NPV of $99-100 \%$.

As opposed to ruling out disease, ruling in disease requires high specificity and PPV. Although we identified high-specificity ( $\geq 90 \%$ ) cutoffs, our cohort was assembled from an outpatient setting, where the pre-test probability of advanced fibrosis tends to be low. In this situation, 


\section{Fibrosis Stage 0}
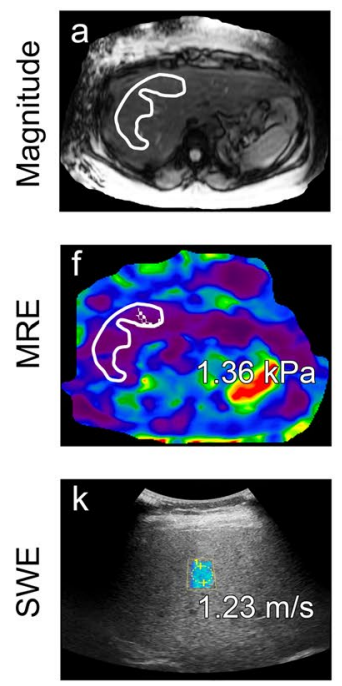

Fibrosis Stage 1
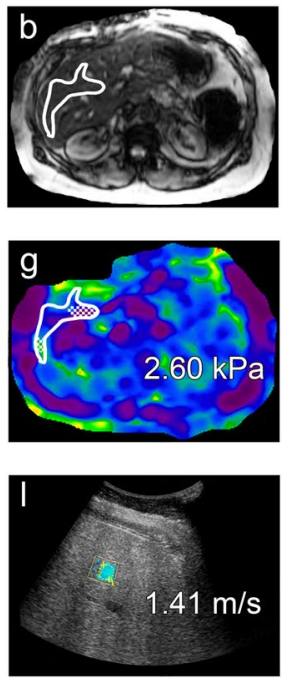
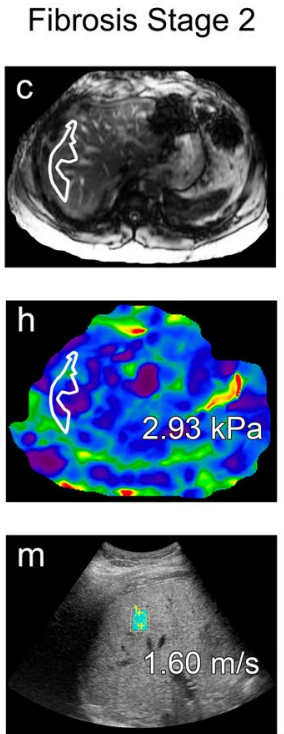

Fibrosis Stage 3
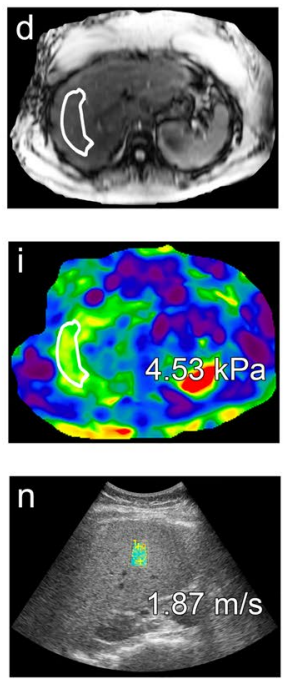

Fibrosis Stage 4
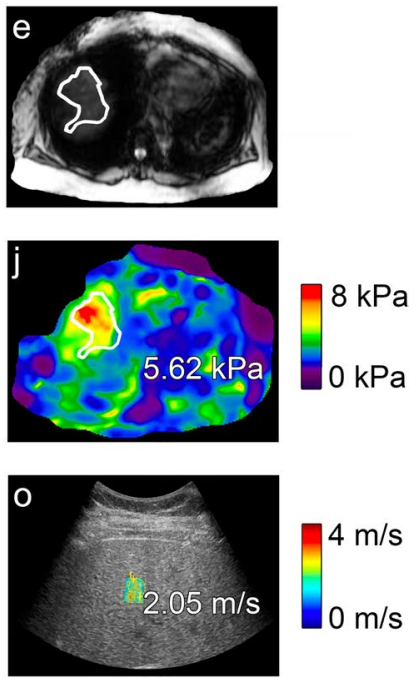

Fig. 3 Transverse colorized MR elastograms (3-T GE 750 scanner using 2D GRE technique, top) and ultrasound-based SWE images with colorized elasticity in the ROIs (GE Logiq E9 with C1-6 transducer, bottom) demonstrate increasing shear stiffness estimates $(\mathrm{kPa})$ or shear wave speed estimates $(\mathrm{m} / \mathrm{s})$ as histologically determined liver fibrosis stage (Nonalcoholic Steatohepatitis Clinical Research Network) increases in patients with nonalcoholic fatty liver disease. From left to right: stage 0 in a 46 -year-old woman; stage 1 in a 56-year-old woman; stage 2 in a 44-year-old man; stage 3 in a

despite applying high-specificity cutoffs, the PPVs for ruling in advanced fibrosis (62\% PPV for MRE, 56\% PPV for SWE) are not sufficient to avoid biopsy altogether. Our results are consistent with those reported by Loomba et al., where an MRE stiffness cutoff of $3.63 \mathrm{kPa}$ yielded a specific result (91\%) and a high NPV of $97 \%$ for excluding stage $\geq 3$ fibrosis in NAFLD patients, but a PPV of only $68 \%$ for ruling it in [31]. Furlan et al.reported similar MRE stiffness cutoff of $3.4 \mathrm{kPa}$ for excluding stage $\geq 3$ fibrosis with a specificity of $91.7 \%$, but a lower NPV of $91.7 \%$ and a much higher PPV of $87 \%$ compared to our results [10]. The higher prevalence of stage $\geq 3$ fibrosis in Furlan et al. compared to this study (39\% versus 16\%) contributed at least in part to the differences in reported NPV and PPV. Thus, if confirmation of advanced fibrosis is desired, then further evaluation possibly including a liver biopsy may be needed. Combining noninvasive tests with clinical decision support tools such as the NAFLD fibrosis score or the FIB-4 test might also improve the PPV [32, 33].

Our study has several limitations. First, the small sample sizes of obese and nonobese subsets as well as the nonuniform stratification of steatosis severity by PDFF cutoff values limited our assessment of obesity and steatosis and their confounding effects on SWE and MRE. Future studies are 42-year-old woman; stage 4 in a 68 -year-old woman. Magnitude of complex modulus in $\mathrm{kPa}$, ROIs, and an automated confidence grid set to $95 \%$ are overlain on the MR elastograms. ROIs depicted in the MRE imaging examples for stages $0,1,2,3$, and 4 are $158 \mathrm{~cm}^{2}, 63$ $\mathrm{cm}^{2}, 80 \mathrm{~cm}^{2}, 90 \mathrm{~cm}^{2}$, and $126 \mathrm{~cm}^{2}$, respectively. Shear wave speed estimates are overlain on the SWE images. ROIs depicted in the SWE imaging examples for stages $0,1,2,3$, and 4 are $1.2 \mathrm{~cm}^{2}, 0.7 \mathrm{~cm}^{2}, 0.9$ $\mathrm{cm}^{2}, 1.1 \mathrm{~cm}^{2}$, and $0.9 \mathrm{~cm}^{2}$, respectively

needed to verify our preliminary finding from the exploratory analyses that MRE is superior to SWE regardless of body habitus and steatosis severity. Also, the distribution of liver fibrosis in our cohort is skewed towards the milder end of the spectrum. Although this may increase the applicability of our results to common clinical contexts such as fibrosis screening, the relatively low number of participants with fibrosis stage $\geq 2$ compared to fibrosis stage $0-1$ limits discrimination between adjacent advanced fibrosis stages. For instance, for a predefined sensitivity $\geq 90 \%$, MRE cutoff is the same for fibrosis stages 2,3 , and $4(2.77 \mathrm{kPa})$ while SWE cutoff for fibrosis stage 3 is lower than for fibrosis stage 2. For the purposes of comparing SWE and MRE, fibrosis distribution affects both techniques equally and would not introduce a bias in favor of one method. Second, this study was conducted using US and MRI systems from a single manufacturer at a single subspecialty center focused on NAFLD research, which may limit the generalizability of its results to other settings such as community centers or sites with systems from other vendors. Third, as technology advances rapidly, it is possible that newer technologies would have provided more accurate performance. For SWE, this might include the use of software that provides realtime feedback on the quality of shear wave propagation and the use of time-harmonic elastography techniques in obese 
Table 2 AUCs and AUC comparisons for SWE and MRE by dichotomized fibrosis stage

\begin{tabular}{|c|c|c|c|c|}
\hline Method & Fibrosis stage 0 vs. $1-4$ & Fibrosis stage $0-1$ vs. $2-4$ & Fibrosis stage $0-2$ vs. $3-4$ & Fibrosis stage $0-3$ vs. 4 \\
\hline \multicolumn{5}{|c|}{ All participants $(n=100)$} \\
\hline & Stage $0=43 ;$ stage $1-4=57$ & Stage $0-1=79 ;$ stage $2-4=21$ & Stage $0-2=84$; stage $3-4=16$ & Stage $0-3=94$; stage $4=6$ \\
\hline SWE & $0.65(0.54-0.76)$ & $0.81(0.71-0.91)$ & $0.85(0.74-0.96)$ & $0.91(0.79-1.00)$ \\
\hline MRE & $0.81(0.72-0.89)$ & $0.94(0.89-1.00)$ & $0.95(0.89-1.00)$ & $0.92(0.83-1.00)$ \\
\hline$p^{\mathrm{a}}$ & $0.005^{*}$ & $0.009 *$ & 0.053 & 0.720 \\
\hline \multicolumn{5}{|c|}{ Subset of obese participants $(n=64)$} \\
\hline & Stage $0=24 ;$ stage $1-4=40$ & Stage $0-1=48$; stage $2-4=16$ & Stage $0-2=51$; stage $3-4=13$ & Stage $0-3=59 ;$ stage $4=5$ \\
\hline SWE & $0.65(0.51-0.79)$ & $0.81(0.69-0.92)$ & $0.83(0.70-0.96)$ & $0.90(0.75-1.00)$ \\
\hline MRE & $0.84(0.74-0.94)$ & $0.92(0.85-1.00)$ & $0.93(0.87-1.00)$ & $0.90(0.78-1.00)$ \\
\hline$p^{\mathrm{a}}$ & $0.008^{*}$ & 0.056 & 0.093 & 0.972 \\
\hline \multicolumn{5}{|c|}{ Subset of nonobese participants $(n=36)$} \\
\hline & Stage $0=20 ;$ stage $1-4=16$ & Stage $0-1=31 ;$ stage $2-4=5$ & Stage $0-2=33$; stage $3-4=3$ & Stage $0-3=35$; stage $4=1$ \\
\hline SWE & $0.66(0.48-0.85)$ & $0.85(0.62-1.00)$ & $0.99(0.96-1.00)$ & NA \\
\hline MRE & $0.75(0.58-0.92)$ & $0.99(0.98-1.00)$ & $1.00(1.00-1.00)$ & NA \\
\hline$p^{\mathrm{a}}$ & 0.354 & 0.214 & 0.480 & NA \\
\hline \multicolumn{5}{|c|}{ Subset of participants with moderate-to-severe steatosis $\left(\mathrm{PDFF}^{\mathrm{a}} \geq 17.43 \%\right)(n=32)$} \\
\hline & Stage $0=10 ;$ stage $1-4=22$ & Stage $0-1=27 ;$ stage $2-4=5$ & Stage $0-2=29 ;$ stage $3-4=3$ & Stage $0-3=31 ;$ stage $4=1$ \\
\hline SWE & $0.59(0.34-0.83)$ & $0.77(0.57-0.97)$ & $0.74(0.41-1.00)$ & NA \\
\hline MRE & $0.83(0.68-0.98)$ & $0.81(0.63-1.00)$ & $0.82(0.60-1.00)$ & NA \\
\hline$p^{\mathrm{a}}$ & $0.024 *$ & 0.655 & 0.424 & NA \\
\hline \multicolumn{5}{|c|}{ Subset of participants with none-to-mild steatosis $\left(\operatorname{PDFF}^{\mathrm{a}}<17.43 \%\right)(n=68)$} \\
\hline & Stage $0=33 ;$ stage $1-4=35$ & Stage $0-1=52$; stage $2-4=16$ & Stage $0-2=55$; stage $3-4=13$ & Stage $0-3=63$; stage $4=5$ \\
\hline SWE & $0.72(0.59-0.84)$ & $0.82(0.70-0.95)$ & $0.88(0.76-0.99)$ & $0.96(0.92-1.00)$ \\
\hline MRE & $0.82(0.72-0.92)$ & $0.98(0.96-1.00)$ & $0.98(0.95-1.00)$ & $0.96(0.90-1.00)$ \\
\hline$p^{\mathrm{a}}$ & 0.127 & $0.009 *$ & 0.075 & 0.883 \\
\hline
\end{tabular}

$A U C$ area under the receiver operating characteristic curve, $S W E$ shear wave elastography, $M R E$ magnetic resonance elastography, $P D F F$ protondensity fat fraction; $95 \%$ confidence interval in parenthesis

${ }^{a}$ Mean proton-density fat fraction (\%) of 9 liver segments

${ }^{*} p$ value, as calculated by DeLong's test to compare the AUCs of MRE and SWE. Using Bonferroni correction, individual $p$ value $<0.05 / 3$ (for grouped AUC, sensitivity, and specificity) is considered significant (asterisk)

Table 3 Diagnostic performance of SWE and MRE at classifying dichotomized fibrosis stages for predefined sensitivity $\geq 90 \%$

\begin{tabular}{llllllllll}
\hline Fibrosis stage & $N$ positive & $N$ negative & Method & Cutoff & Sensitivity & Specificity & PPV & NPV & Accuracy \\
\hline Stage 1-4 vs. 0 & \multirow{2}{*}{57} & 43 & MRE & $2.01 \mathrm{kPa}$ & 0.912 & $0.488^{*}$ & 0.703 & 0.808 & 0.730 \\
& & & SWE & $1.27 \mathrm{~m} / \mathrm{s}$ & 0.912 & 0.116 & 0.578 & 0.500 & 0.570 \\
Stage 2-4 vs. 0-1 & \multirow{2}{*}{21} & \multirow{2}{*}{49} & MRE & $2.77 \mathrm{kPa}$ & 0.905 & $0.848^{*}$ & 0.613 & 0.971 & 0.860 \\
& & & SWE & $1.49 \mathrm{~m} / \mathrm{s}$ & 0.905 & 0.430 & 0.297 & 0.944 & 0.530 \\
Stage 3-4 vs. 0-2 & \multirow{2}{*}{16} & \multirow{2}{*}{84} & MRE & $2.77 \mathrm{kPa}$ & 0.938 & $0.810^{*}$ & 0.484 & 0.986 & 0.830 \\
& & & SWE & $1.46 \mathrm{~m} / \mathrm{s}$ & 0.938 & 0.393 & 0.227 & 0.971 & 0.480 \\
Stage 4 vs. 0-3 & \multirow{2}{*}{6} & \multirow{2}{*}{94} & MRE & $2.77 \mathrm{kPa}$ & 1.000 & 0.734 & 0.194 & 1.000 & 0.750 \\
& & & SWE & $1.59 \mathrm{~m} / \mathrm{s}$ & 1.000 & 0.617 & 0.143 & 1.000 & 0.640 \\
\hline
\end{tabular}

$M R E$ magnetic resonance elastography, $S W E$ shear wave elastography, $P P V$ positive predictive value, $N P V$ negative predictive value; $k P a$ kilopascals, unit for shear stiffness as measured by MRE; $m / s$ meters per second, unit for shear wave speed as measured by SWE

*Specificity of MRE is significantly higher than that of SWE based on two-tailed McNemar's test, $p<0.001$. Using Bonferroni correction, individual $p$ value $<0.05 / 3$ (for grouped AUC, sensitivity, and specificity) is considered significant 
Table 4 Diagnostic performance of SWE and MRE at classifying dichotomized fibrosis stages for predefined specificity $\geq 90 \%$

\begin{tabular}{llllllllll}
\hline Fibrosis stage & $N$ positive & $N$ negative & Method & Cutoff & Sensitivity & Specificity & PPV & NPV & Accuracy \\
\hline Stage 1-4 vs. 0 & \multirow{2}{*}{57} & \multirow{2}{*}{43} & MRE & $2.60 \mathrm{kPa}$ & $0.579^{*}$ & 0.907 & 0.892 & 0.619 & 0.720 \\
& & & SWE & $1.75 \mathrm{~m} / \mathrm{s}$ & 0.333 & 0.907 & 0.826 & 0.506 & 0.580 \\
Stage 2-4 vs. 0-1 & \multirow{2}{*}{21} & \multirow{2}{*}{0} & MRE & $3.06 \mathrm{kPa}$ & $0.810^{*}$ & 0.911 & 0.708 & 0.947 & 0.890 \\
& & & SWE & $1.79 \mathrm{~m} / \mathrm{s}$ & 0.476 & 0.911 & 0.588 & 0.867 & 0.720 \\
Stage 3-4 vs. 0-2 & \multirow{2}{*}{16} & \multirow{2}{*}{84} & MRE & $3.17 \mathrm{kPa}$ & 0.813 & 0.905 & 0.619 & 0.962 & 0.890 \\
& \multirow{2}{*}{6} & \multirow{2}{*}{94} & SWE & $1.78 \mathrm{~m} / \mathrm{s}$ & 0.625 & 0.905 & 0.556 & 0.927 & 0.860 \\
Stage 4 vs. 0-3 & \multirow{2}{*}{} & & MRE & $3.42 \mathrm{kPa}$ & 0.667 & 0.904 & 0.308 & 0.977 & 0.890 \\
& & & SWE & $1.81 \mathrm{~m} / \mathrm{s}$ & 0.833 & 0.904 & 0.357 & 0.988 & 0.900 \\
\hline
\end{tabular}

$M R E$ magnetic resonance elastography, $S W E$ shear wave elastography, $P P V$ positive predictive value, $N P V$ negative predictive value; $k P a$ kilopascals, unit for shear stiffness as measured by MRE; $\mathrm{m} / \mathrm{s}$ meters per second, unit for shear wave speed as measured by SWE

*Sensitivity of MRE is significantly higher than that of SWE based on two-tailed McNemar's test, $p \leq 0.01$. Using Bonferroni correction, individual $p$ value $<0.05 / 3$ (for grouped AUC, sensitivity, and specificity) is considered significant

patients. For MRE, this might include a spin-echo echo planar imaging sequence rather than a GRE sequence and/or the use of thin flexible blanket-like torso phased array coils. Finally, we did not test the longitudinal reproducibility of the two modalities, a factor that would be important for determining the best test for monitoring treatment response. The comparative performance of SWE and MRE for longitudinal monitoring remains a gap in knowledge for which future research is needed.

In conclusion, this prospective study provided direct comparison of SWE versus MRE for staging fibrosis in a cohort of participants with known or suspected NAFLD and clinically indicated liver biopsy. We showed that in patients in whom both methods are adequate, MRE had significantly higher accuracy than SWE for diagnosing earlier $(\geq 1$ and $\geq 2$ ) fibrosis stages. For purposes of directing biopsy to detect advanced fibrosis, SWE and MRE performed equally well, both demonstrating high NPV for excluding disease. Future studies that aim to evaluate the relative reproducibility of these modalities for longitudinal monitoring and the cost-effectiveness of various diagnostic approaches using combinations of SWE, MRE, biopsy, and clinical decision support will further inform optimal usage of both methods for clinical care and clinical trials.

Supplementary Information The online version contains supplementary material available at https://doi.org/10.1007/s00330-021-08369-9.

Acknowledgements The authors wish to thank GE Healthcare who provides research support to University of California, San Diego. This work has been supported by the following grants: National Institutes of Health (NIH) Grants: R01DK088925, R01DK106419.

Funding Activities related to the present article: Institution received a grant from General Electric.

\section{Declarations}

Guarantor The scientific guarantor of this publication is Dr. Claude B. Sirlin.

Conflict of interest The authors of this manuscript declare relationships with the following companies, for which the authors engaged in activities not related to the present article: RL serves as a consultant or advisory board member for Arrowhead Pharmaceuticals, AstraZeneca, Bird Rock Bio, Boehringer Ingelheim, Bristol-Myers Squibb, Celgene, Cirius, CohBar, Conatus, Eli Lilly, Galmed, Gemphire, Gilead, Glympse bio, GNI, GRI Bio, Intercept, Ionis, Janssen, Merck, Metacrine, NGM Biopharmaceuticals, Novartis, Novo Nordisk, Pfizer, Prometheus, Sanofi, Siemens, and Viking Therapeutics. CBS is on the advisory boards of AMRA, Guerbet, and VirtualScopics; is a consultant for GE Healthcare, Bayer, Boehringer Ingelheim, AMRA, and Fulcrum; institution received grants from Gilead, GE Healthcare, Siemens, GE MRI, Bayer, GE Digital, GE Ultrasound, ACR Innovation, and Philips; served on the speakers bureau for GE Healthcare; developed educational presentations for Medscape and Resoundant; institution has lab service agreements with Enanta, ICON, Gilead, Shire, VirtualScopics, Intercept, Synageva, Takeda, Genzyme, Janssen, and NuSirt.

Statistics and biometry Tanya Wolfson, MA, kindly provided statistical advice for this manuscript and is one of the authors.

Informed consent Written informed consent was obtained from all patients in this study.

Ethical approval Institutional Review Board approval was obtained.

\section{Methodology}

-Cross-sectional study

Open Access This article is licensed under a Creative Commons Attribution 4.0 International License, which permits use, sharing, adaptation, distribution and reproduction in any medium or format, as long as you give appropriate credit to the original author(s) and the source, provide a link to the Creative Commons licence, and indicate if changes were made. The images or other third party material in this article are included in the article's Creative Commons licence, unless indicated otherwise in a credit line to the material. If material is not included in 
the article's Creative Commons licence and your intended use is not permitted by statutory regulation or exceeds the permitted use, you will need to obtain permission directly from the copyright holder. To view a copy of this licence, visit http://creativecommons.org/licenses/by/4.0/.

\section{References}

1. Younossi ZM, Koenig AB, Abdelatif D et al (2016) Global epidemiology of nonalcoholic fatty liver disease-meta-analytic assessment of prevalence, incidence, and outcomes. Hepatology 64:73-84. https://doi.org/10.1002/hep.28431

2. Hagström H, Nasr P, Ekstedt M et al (2017) Fibrosis stage but not NASH predicts mortality and time to development of severe liver disease in biopsy-proven NAFLD. J Hepatol 67:1265-1273. https://doi.org/10.1016/j.jhep.2017.07.027

3. Musso G, Cassader M, Paschetta E, Gambino R (2017) Thiazolidinediones and advanced liver fibrosis in nonalcoholic steatohepatitis. JAMA Intern Med 177:633. https://doi.org/10.1001/jamai nternmed.2016.9607

4. Loomba R, Lawitz E, Mantry PS et al (2018) The ASK1 inhibitor selonsertib in patients with nonalcoholic steatohepatitis: a randomized, phase 2 trial. Hepatology 67:549-559. https://doi.org/10. 1002/hep.29514

5. Tapper EB, Lok AS-F (2017) Use of liver imaging and biopsy in clinical practice. N Engl J Med 377:756-768. https://doi.org/10. 1056/NEJMra1610570

6. Lee MS, Bae JM, Joo SK et al (2017) Prospective comparison among transient elastography, supersonic shear imaging, and ARFI imaging for predicting fibrosis in nonalcoholic fatty liver disease. PLoS One 1-17. https://doi.org/10.1371/journal.pone. 0188321

7. Cassinotto C, Boursier J, de Lédinghen V et al (2016) Liver stiffness in nonalcoholic fatty liver disease: a comparison of supersonic shear imaging, FibroScan, and ARFI with liver biopsy. Hepatology 63:1817-1827. https://doi.org/10.1002/hep.28394

8. Cui J, Heba E, Hernandez C et al (2016) Magnetic resonance elastography is superior to acoustic radiation force impulse for the diagnosis of fibrosis in patients with biopsy-proven nonalcoholic fatty liver disease: a prospective study. Hepatology 63:453-461. https://doi.org/10.1002/hep.28337

9. Singh S, Venkatesh SK, Loomba R et al (2016) Magnetic resonance elastography for staging liver fibrosis in non-alcoholic fatty liver disease: a diagnostic accuracy systematic review and individual participant data pooled analysis. Eur Radiol 26:1431-1440. https://doi.org/10.1007/s00330-015-3949-z

10. Furlan A, Tublin ME, Yu L et al (2020) Comparison of 2D shear wave elastography, transient elastography, and MR elastography for the diagnosis of fibrosis in patients with nonalcoholic fatty liver disease. AJR Am J Roentgenol 214:W20-W26. https://doi. org/10.2214/AJR.19.21267

11. Imajo K, Honda $Y$, Kobayashi $T$ et al (2020) Direct comparison of US and MR elastography for staging liver fibrosis in patients with nonalcoholic fatty liver disease. Clin Gastroenterol Hepatol. https://doi.org/10.1016/J.CGH.2020.12.016

12. Kleiner DE, Brunt EM, Van Natta M et al (2005) Design and validation of a histological scoring system for nonalcoholic fatty liver disease. Hepatology 41:1313-1321. https://doi.org/10.1002/ hep. 20701

13. Dietrich C, Bamber J, Berzigotti A et al (2017) EFSUMB guidelines and recommendations on the clinical use of liver ultrasound elastography, update 2017 (long version). Ultraschall Med 38:e16-e47. https://doi.org/10.1055/s-0043-103952

14. Committee USB (2017) QIBA SWS profile checklist for fibrosis 2017. In: Ultrasound SWS Biomark. Comm. http://qibawiki.
rsna.org/index.php/Ultrasound_SWS_Biomarker_Ctte. Accessed 8 Nov 2018

15. Wagner M, Corcuera-Solano I, Lo G et al (2017) Technical failure of MR elastography examinations of the liver: experience from a large single-center study. Radiology 284:401-412. https://doi.org/ 10.1148/radiol.2016160863

16. Yin M, Talwalkar JA, Glaser KJ et al (2007) Assessment of hepatic fibrosis with magnetic resonance elastography. Clin Gastroenterol Hepatol 5:1207-1213.e2. https://doi.org/10.1016/j.cgh. 2007.06.012

17. Dzyubak B, Glaser K, Yin M et al (2013) Automated liver stiffness measurements with magnetic resonance elastography. J Magn Reson Imaging 38:371-379. https://doi.org/10.1002/jmri.23980

18. Yin M, Glaser KJ, Talwalkar JA et al (2016) Hepatic MR elastography: clinical performance in a series of 1377 consecutive examinations. Radiology 278:114-124. https://doi.org/10.1148/ radiol.2015142141

19. Park CC, Nguyen P, Hernandez C et al (2017) Magnetic resonance elastography vs transient elastography in detection of fibrosis and noninvasive measurement of steatosis in patients with biopsy-proven nonalcoholic fatty liver disease. Gastroenterology 152:598-607.e2. https://doi.org/10.1053/j.gastro.2016.10.026

20. Jayakumar S, Middleton MS, Lawitz EJ et al (2019) Longitudinal correlations between MRE, MRI-PDFF, and liver histology in patients with non-alcoholic steatohepatitis: Analysis of data from a phase II trial of selonsertib. J Hepatol 70:133-141. https://doi. org/10.1016/j.jhep.2018.09.024

21. Tang A, Desai A, Hamilton G et al (2015) Accuracy of MR imaging-estimated proton density fat fraction for classification of dichotomized histologic steatosis grades in nonalcoholic fatty liver disease. Radiology 274:416-425. https://doi.org/10.1148/ radiol.14140754

22. Tang A, Tan J, Sun M et al (2013) Nonalcoholic fatty liver disease: MR imaging of liver proton density fat fraction to assess hepatic steatosis. Radiology 267:422-431. https://doi.org/10.1148/radiol. 12120896

23. Loomba R, Sirlin CB, Ang B et al (2015) Ezetimibe for the treatment of nonalcoholic steatohepatitis: assessment by novel magnetic resonance imaging and magnetic resonance elastography in a randomized trial (MOZART trial). Hepatology 61:1239-1250. https://doi.org/10.1002/hep.27647

24. Middleton MS, Heba ER, Hooker CA et al (2017) Agreement between magnetic resonance imaging proton density fat fraction measurements and pathologist-assigned steatosis grades of liver biopsies from adults with nonalcoholic steatohepatitis. Gastroenterology 153:753-761. https://doi.org/10.1053/j.gastro.2017.06. 005

25. Xiao G, Zhu S, Xiao X et al (2017) Comparison of laboratory tests, ultrasound, or magnetic resonance elastography to detect fibrosis in patients with nonalcoholic fatty liver disease: a metaanalysis. Hepatology 66:1486-1501. https://doi.org/10.1002/hep. 29302

26. Herrmann E, de Lédinghen V, Cassinotto C et al (2018) Assessment of biopsy-proven liver fibrosis by two-dimensional shear wave elastography: an individual patient data-based meta-analysis. Hepatology 67:260-272. https://doi.org/10.1002/hep.29179

27. Yoon JH, Lee JM, Joo I et al (2014) Hepatic fibrosis: prospective comparison of MR elastography and US shear-wave elastography for evaluation. Radiology 273:772-782. https://doi.org/10.1148/ radiol. 14132000

28. Calvaruso V, Burroughs AK, Standish R et al (2009) Computerassisted image analysis of liver collagen: relationship to Ishak scoring and hepatic venous pressure gradient. Hepatology 49:1236-1244. https://doi.org/10.1002/hep.22745

29. Chen J, Yin M, Talwalkar JA et al (2017) Diagnostic performance of MR elastography and vibration-controlled transient 
elastography in the detection of hepatic fibrosis in patients with severe to morbid obesity. Radiology 283:418-428. https://doi.org/ 10.1148/radiol.2016160685

30. Chalasani N, Younossi Z, Lavine JE et al (2018) The diagnosis and management of nonalcoholic fatty liver disease: practice guidance from the American Association for the Study of Liver Diseases. Hepatology 67:328-357. https://doi.org/10.1002/hep. 29367

31. Loomba R, Wolfson T, Ang B et al (2014) Magnetic resonance elastography predicts advanced fibrosis in patients with nonalcoholic fatty liver disease: a prospective study. Hepatology. https:// doi.org/10.1002/hep. 27362
32. Jung J, Loomba RR, Imajo K et al (2020) MRE combined with FIB-4 (MEFIB) index in detection of candidates for pharmacological treatment of NASH-related fibrosis. Gut. https://doi.org/ 10.1136/gutjnl-2020-322976

33. Angulo P, Hui JM, Marchesini G et al (2007) The NAFLD fibrosis score: a noninvasive system that identifies liver fibrosis in patients with NAFLD. Hepatology 45:846-854. https://doi.org/10.1002/ hep. 21496

Publisher's Note Springer Nature remains neutral with regard to jurisdictional claims in published maps and institutional affiliations. 